\title{
Aspects of Infection in Intensive Care Unit - Prevention and Control
}

\author{
MK Hassan ${ }^{1}$, H Akhter ${ }^{2}$, MMG Chowdhury ${ }^{1}$, N Haq ${ }^{3}$, NAK Ahsan ${ }^{1}$ \\ ${ }^{1}$ Department of Cardiovascuar Surgery, NICVD, ${ }^{2}$ Department of Gynae \& Obs, CMH, Dhaka, \\ ${ }^{3}$ Department of Anaesthesia NICVD, Dhaka
}

\begin{abstract}
Key words:
Intensive care unit,

Pneumonia, ventilator pneumonia
\end{abstract}

\begin{abstract}
:
Intensive Care Unit (ICU) is the most vulnerable area in the hospital premises. Hospital acquired infections in critically ill patients as well as of transmission of infections from a patient to another patient or to the health care workers or vice versa can occur in ICU. ICUs combine physicians, nurses and allied health professionals to manage patients with life threatening single or multiple organ system failure, including stabilization after surgical interventions. It is a continuous management including monitoring, diagnosis, and support of failing vital functions as well as the treatment of the underlying disease. Here we have to discuss the source of infection and their effective control measurement in intensive care unit. Ventilator associated pneumonia (VAP) as well with other sources is most common nosocomial infection having $33 \%$ mortality in ICU.
\end{abstract}

(CVJ 2008; 1(1) : 78-80)

\section{Introduction:}

Intensive Care Units (ICU) are the most expensive part of a hospital, they are used in most efficient way. High quality and cost-effective performance in intensive care medicine (ICM) can best be achieved when responsibility and management are given to those who have the special expertise. ${ }^{1}$ In western Europe ICUs, 72\% committed 24-hour doctor are on duty.

In ICU, hospital acquired infection can be minimized by prevention of transmission of infection from a patient to another patient or to the health care workers or vice versa.

Effective control of infection in ICU involves a rational and appropriate use of antibiotics to treat the infection and prevent emergence of resistant strains of microorganisms. $^{2}$

\section{Sources of Infection:}

Sources of infection in ICU are multivariate. The most vulnerable sources are discussed herewith:

Ventilator-associated Pneumonia (VAP) is an important part of all nosocomial infections in ICU is a serious illness with substantial morbidity and mortality and increases costs of hospital care. Respiratory failure, coma, depressed consciousness, enteral feeding and length of stay are independent risk factors for developing VAP. The cost of VAP is approximately five fold higher than non-infected patients. ${ }^{3}$ This nosocomial pneumonia is the most common nosocomial infection in ICU; the prevalence is $47 \%$ with an attributive mortality of $33 \% .{ }^{4}$ In intubated and mechanically ventilated patients the risk of acquiring ventilator-associated pneumonia is $1 \%$ per day on the ventilator. ${ }^{2}$

Acquired bloodstream infection (BSI) in the ICU is a serious complication. A study of ICU patients in Calgary demonstrated crude death rates of $45 \%$ among patients with ICU-acquired BSI as compared with $21 \%$ in those without. ${ }^{5}$

Sepsis is one of the most common causes of death in the world with a mortality rate of approximately 29\%. ${ }^{6}$ The overall 30 -day mortality in the ICU is typically $20 \%$. The 30 day mortality in the population with severe sepsis, defined as sepsis with organ dysfunction, is $30-50 \% .{ }^{6}$ Sepsis was diagnosed in $58 \%$ of the patients in 101 consecutive critically ill patients. the principal site of infection was the lung. Microorganisms isolated were Strep. pneumonie mostly and then Pseudomonas aeroginosa, Staph. aureus, and etc. ${ }^{7}$

Anaemic Syndrome: It is a frequent problem in ICU, probably etiology is the suppression of the erythropoietin response due to the direct effects 
of cytokines, as well as frequent blood sampling. Transfusion is not free of complication; therefore transfusion reactions are estimated to occur in $2 \%$ of the total packed cells transfused. This transfusion practice is an independent risk factor for the development of nosocomial pneumonia. ${ }^{4}$

Thrombocytopenia: Thrombocytopenia of less than $50 \times 10^{9} / \mathrm{L}$ platelets may be a marker for more severe illness and increased risk of death, rather than causative, because a true causal relationship is not established. Thrombocytopenia also leads to an excess of blood product consumption. ${ }^{8}$ In the absence of neutropenia, thrombocytopenia has been positively correlated with an increased incidence and severity of pneumonia in elderly patients. ${ }^{8}$

Inadequate splanchic perfusion in critically ill patient is associated with increased morbidity and mortality. In endotoxin induced septicemia, gut blood flow becomes very low and no drugs are yet available that increase total splanchnic blood flow selectively and to a clinically relevant extent. ${ }^{9}$ Infection caused in abdominal organ (gastrointestinal tract, liver, bile duct and pancreas) is mostly by Clostridium dificilleassociated colitis. ${ }^{7}$

Urinary tract infection (UTI): Patients in the ICU are commonly catheterized. the catheterization introduces the perineal flora into the urinary bladder and interferes with the natural defence mechanisms of the bladder and urethra to prevent colonization of urine. In UTI, E. coli, Pseudomonas are mostly isolated. ${ }^{7}$

Infection with intravascular devices: Central lines are associated with serious morbidity and mortality due to infections. Risk of sepsis with triple lumen catheters used for parenteral nutrition and medication is four times that of single channel catheters. ${ }^{2}$

\section{Precautions of Infection Control:}

$V A P$ : The ventilator itself is not a source of microorganism and filters should be placed between the circuit and machine to protect it. However, the condensate that collects in the breathing circuit of the humidified system rapidly becomes colonized with bacteria from the patients' oropharynx. Therefore, this condensate should not be allowed to drain into the patient's respiratory tract or humidifier but periodically drained out. Staff handling the circuits may easily acquire the bacteria in their hands and in turn transmit them to other patients; therefore gloves should be worn and hands should be washed after ventilator tubing is handled. The circuits are decontaminated by washing but thorough drying is essential. Similarly, humidifiers and nebulizers should use only sterile water and should be washed and dried after each use. All other respiratory therapy equipment should also be washed and dried periodically i.e. T-piece, reservoir bags, etc. ${ }^{2}$

Decontamination of equipment: Heavily contaminated equipment e.g. suction jar, bedpans should be routinely decontaminated every day. In the ICU, where suction is used frequently, a disposable suction system may be used to avoid decontaminating suction jars.

Hand washing: Skin is normally dry, acidic and poor in nutrients, therefore in hospitable for most organisms. Some gram-positive organisms are present in deep skin cervices e.g. coagulase negative Staphylococci, micrococcioid, coryneforms. These 'resident flora' are not removed by simple hand washing but their load can be decreased by a combination of soap and a microbicide e.g. chlorhexidine or povidone-iodine, specially if used repeatedly.

Intravascular defence: Staph. aureus is isolated mostly from infection related to intravascular devices. In this context, the development of an antistaphylococcal vaccine (staph vax) represents a promising new health technology.

UTI: In order to prevent catheter-associated infections, strict asepsis should be maintained during insertion and use. The insertion site should be kept clean and dressed. The key practices for preventing catheter-associated infection are minimizing the duration of catheterization and ensuring that closed drainage system remains closed. A clean pair of gloves should be worn for emptying urine bags and discarded after use.

Steroid therapy: Severe sepsis and septic shock patient should receive early aggressive therapy to restore and maintain oxygen availability to the cells. There should also be generous use of fluids and inotropic agents titrated by appropriate hemodynamic monitoring. 
Administration of moderate dose corticosteroid should be considered in cases of refractory septic shock, particularly in those with relative adrenal insufficiency. It is recommended that an ACTH test can be carried out before starting the intervention. ${ }^{6}$ The steroid therapy group received prednisolone at a physiological dose. Prednisolone should be given intravenously at 6: $00(5 \mathrm{mg})$ and 18: $00(2.5 \mathrm{mg})$ for 10 days. ${ }^{10}$

Antibiotic therapy: Rigorous adherence to good infection control practice is essential to control infection in ICU. In addition, rational use of antibiotics also aids in preventing the spread of multi-drug resistant microbes. Till culture sensitivities are available, antibiotics should be prescribed depending on bacterial ecology of the unit. The specific antibiotics should be given once sensitivities are available. Disregard positive cultures from nonsterile sites, which represent colonization with non-pathogens. Without draining abscess or collections antibiotics will be ineffective.

\section{Conclusion:}

At Waikat of Hospital, New Zealand, a total of 5916 individual patients were admitted for ICU care between 1991 and 1997. Of them, 525 (9\%) died in ICU. Most of the deaths occured included hypovolaemia resulting renal failure, fluid overload leading to pulmonary oedema, delay in starting antibiotics despite proven infection, drug omission and inappropriate arrhythmia management, poor blood pressure control resulting in cardiac arrest and prolonged hypoxia resulting in respiratory arrest. ${ }^{11}$

Hyperglycaemia, caused by insulin resistance in the liver and muscle, is a common finding in ICU patients. It can be considered an adaptive response, providing glucose for wound healing. ${ }^{6}$
Intensive care units combine physicians, nurses and allied health professionals to manage patients with life threatening single or multiple organ system failure, including stabilization after surgical interventions. It is a continuous $(24 \mathrm{hr})$ management including monitoring, diagnosis, support of failing vital functions, as well as the treatment of the underlying disease. ${ }^{1}$

\section{Reference:}

1. Hilmar B, Onnen M. 'Twenty four hour presence of physicians in the ICU.' Critical Care 2001; 5: 131-137.

2. Kaul HL, Chhabra A. 'Aspects of infection control in intensive care units.' Seminar of $5^{\text {th }}$ South Asian Conference of Anaesthesiologists 2003; V.T. pp 99-102.

3. Riza HE, Ata NY et al. 'Cost and risk factors for ventilator-associated pneumonia in a Turkish university hospital's intensive care unit: A case-control study.' BMC Pulmonary Medicine 2004; 4 (3): pp 1-7.

4. Yepes D, Gil B et al. 'Ventilator-associated pneumonia and transfusion, Is there really an association (the NAVTRA study).' BMC Pulmonary Medicine 2006; 6 (18): pp 1-5.

5. Suntharalinjam G, Cousins J et al. 'Scanning the horizon: emerging hospital-wide technologies and their impact on critical care.' Critical Care 2005; 9: pp 12-15.

6. Vincent JI, Abraham E. 'Reducing mortality in sepsis: New directions.' Critical Care 2002; 6 (Suppl-3): pp S1S8.

7. Crain MC, Morgenthaler GN et al. 'Mid-regional proadrenomedullin as a prognostic marker in sepsis: an observational study.' Critical Care 2005; 9: R816-R824.

8. Stephan F, J DM et al. 'Thrombocytopenia in critically ill surgical patients: a case-control study evaluating attributable mortality and transfusion requirements.' Critical Care 1999; 3: pp 151-158.

9. Jakob SM. 'Clinical review: splanchnic ischemia.' Critical Care 2002; 6: pp 306-312.

10. Orhan Y, Mehnet D et al. 'Physiological dose steroid therapy in sepsis.' Critical Care 2002; 6: pp 251-258.

11. Laurence A and Havil JH. 'An audit of deaths occurring in hospital after discharge from the intensive care unit.' Anesth Intens Care 1999; 27: pp 185-189. 